\begin{tabular}{|c|c|c|}
\hline Beitr. Ent. & Berlin & ISSN 0005-805X \\
\hline $\mathbf{5 0}(2000) 1$ & S. $65-74$ & 11.04 .2000 \\
\hline
\end{tabular}

\title{
Zwei neue habituell abweichende Arten der Gattung Lesteva LATREILLE, 1796 aus Sibirien
}

\author{
(Coleoptera, Staphylinidae, Omaliinae)
}

Mit 6 Figuren

\section{LOTHAR ZERCHE}

\section{Summary}

Lesteva brathinoides $\mathbf{s p .}$. from the eastern Sajan mountains and Lesteva sajanensis sp. n. from the western Sajan mountains are described and compared with related Lesteva species and with somewhat similar, but not closely related Brathinus species. The facies and the male genitalia of the new species are figured. The systematic positions of the new species within Lesteva are discussed but remain unclear.

\section{Zusammenfassung}

Lesteva brathinoides $\mathbf{s p .} \mathbf{n}$. aus dem östlichen Sajan-Gebirge und Lesteva sajanensis $\mathbf{s p .} \mathbf{n}$. aus dem westlichen Sajan-Gebirge werden beschrieben und mit näher verwandten Lesteva-Arten und etwas ähnlichen, aber nicht näher verwandten Brathinus-Arten verglichen. Habitus und Aedoeagus der neuen Arten werden jeweils abgebildet. Die jeweilige systematische Stellung der neuen Arten innerhalb von Lesteva wird diskutiert, bleibt aber ungeklärt.

\section{Einleitung}

Die Gattung Lesteva, in der Paläarktis mit 75 Arten vertreten, ist in Bezug auf die Länge der Elytren nicht einheitlich. Viele Arten haben kürzere Elytren [z. B. punctata ERICHSON, sicula heeri FAUVEL, benicki LOHSE, gracilis WATANABE, plagiata SHARP]. Bei diesen Arten ist das Abdominaltergit IV sichtbar. Lesteva longoelytrata (GOEZE) hat, im Gegensatz zu ihrem Namen, keine besonders langen Elytren; auch bei ihr ist gewöhnlich das Tergit IV ganz oder teilweise sichtbar. Etwas verlängerte Elytren sind in der Gattung aber weit verbreitet $[\mathrm{z}$. B. bucharica FAUVEL, uhligi ZANETTI, monticola KIESENWETTER, hanseni LOHSE]. Von mehreren Arten kennt man auch eine große Variabilität bzw. Dimorphismus in der Länge der Elytren (LOHSE 1962, ZANETTI 1987).

Keine der bisher bekannten Arten hat aber reduzierte Schultern und sehr gestreckte Elytren, die herab gebogen sind und den Hinterkörper mit dem Abdomen weitgehend einschließen, fast so weit wie bei den Brathinus-Arten.

\section{Danksagung und Erklärung der Abkürzungen zum Typenverbleib}

Mein Dank gebührt Dr. RÜDIGER KRAUSE und OLAF JÄGER, Staatliches Museum für Tierkunde Dresden (MTD) für die Ausleihe der von ihnen gesammelten Art und Dr. WOLFGANG SCHAWALLER, Staatliches Museum für Naturkunde Stuttgart (SMNS), für die Vermittlung der Ausleihe des Materials von Dr. VIKTOR G. SHILENKOV, Irkutsk State University (IGU). 


\section{Lesteva brathinoides $\mathrm{sp} . \mathrm{n}$.}

Fig. 1-3

\section{Typenmaterial}

Holotypus $\left(\sigma^{\top}\right)$ : Sibirien, Ost-Sajan: \Tunkinskije Goltsy, \ westl. Hauptast der $\backslash$ Kyngarga, 3.VII.1993 \\Hochtal zw. 1700 u. $\backslash 2000 \mathrm{~m}$ ü. NN, Schnee- $\backslash$ feldrand u. block- $\backslash$ reicher Steilhang $\backslash$ leg. Krause \& Jäger \Staatl. Museum für \Tierkunde Dresden $\mid$ HOLOTYPUS \ Lesteva $\backslash$ brathinoides $\backslash$ Zerche (MTD).

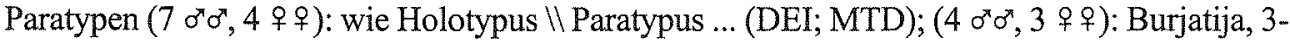
4.VII.93 \Arschan, verch. [= obere] Kyngargi $\backslash 2200-2500 \mathrm{~m}$. \al'pika V. Schilenkov [kyrillisch] II Buryatiya $\backslash$ Arshan, alpine $\backslash$ Zone, Kyngarga valley $\ \backslash$ Paratypus ... (DEI; IGU; SMNS).

\section{Beschreibung}

Maße des Holotypus [in mm]: Kopfbreite 0,76; Kopfmittellänge [ohne Labrum] 0,70; Antennenlänge 2,35; Augenabstand 0,63; Augenlänge 0,17; Schläfenlänge 0,17; Pronotumbreite 1,02 ; Pronotumlänge 0,95 ; Elytrenbreite 1,68; Nahtlänge 1,82; Abdomenbreite 1,54.

Farbe fast einheitlich rotbraun. Kopf im hinteren Teil \pm verdunkelt. Taster, Basis der Antennen und Tarsen gelbrot. Oberseite fein anliegend hell behaart. Punktur des Kopfes gut sichtbar $(40 \mathrm{x})$, die Zwischenräume etwas kleiner als die Punktdurchmesser. Punktur des Pronotums wenig stärker, etwas dichter. Punktur der Elytren deutlich gröber und dichter.

Größe: $\sigma^{x} \sigma^{x}:$ 4,06-5,11 mm (Mittelwert 4,60 mm [n=12]); 우 $:$ 3,85- 4,97 mm (Mittelwert $4,38 \mathrm{~mm}[\mathrm{n}=7])$.

Kopf sehr gestreckt; Clypeus, Labrum und Mandibeln schnabelförmig nach vorn gerichtet; die größte Länge vom Hinterrand bis zur Mandibelspitze deutlich länger als die größte Breite über den Augen (1,26). Scheitel etwas höher als der Hals; Halsfurche oberseits nur angedeutet, unterseits mäßig tief, im Kehlbereich etwas tiefer. Augen klein, nur so lang wie die Schläfen, aber deutlich vorgewölbt. Ocellen vollständig reduziert. Stirngruben vor dem Niveau des Augenvorderrandes, mäßig tief, aber deutlich. Zwischen der sanft abfallenden Stirn und dem nach vorn gerichteten Clypeus eine muldenförmige Vertiefung. Labrum gestreckt. Mandibeln gestreckt trapezförmig, apikal abrupt kurz nach innen gebogen, mit einem spitzen Mittelzahn. Lapialpalpen und Laciniae gestreckt, die Mandibeln überragend. Maxillarpalpen sehr schlank; Proportionen der Segmente [10 entspricht $0,11 \mathrm{~mm}$ ]: I: $5 \times 4$; II: $19 \times 6$; III: $9 \times 5 ; 22 \times 5$. Kehle trapezförmig (Länge : Breite $=2: 2,5$ ). Submentum umgekehrt trapezförmig (Länge : Breite $=2,2: 2,7$ ). Mentum und Submentum fast in einer Ebene liegend.

Antennen sehr lang und schlank, zurückgelegt etwa die Mitte der langen Elytren erreichend. Proportionen der Segmente [10 entspricht 0,11 mm]: I: $20 \times 8,5 ;$ II: $17 \times 6,5 ;$ III: $18 \times 6,5 ;$ IV: $18 \times 7$; V: $22 \times 7$; VI: $20 \times 7$; VII: $20 \times 7$; VIII: $20 \times 8$; IX: $21 \times 9 ;$ X: $20 \times 10 ;$ XI: $32 \times 9$. Segment XI charakteristisch gebaut: in der Mitte ringförmig eingeschnürt, so dass bei flüchtiger Betrachtung der Eindruck von zwei getrennten Segmenten entsteht.

Pronotum im vorderen Drittel am breitesten. Seiten über der breitesten Stelle gleichmäßig gerundet, ohne Andeutung von Vorderwinkeln in den konvexen Vorderrand übergehend; nach hinten deutlich ausgeschweift verengt. Hinterwinkel fast rechtwinklig; die Winkel aber nicht scharf, sondern sehr kurz verrundet. Hinterrand gerade. Seitenrandung vollständig ausgebildet, aber sehr fein, zum Vorderrand bogenförmig verlaufend. Oberfläche querüber stark gewölbt, aber auch in Längsrichtung deutlich gewölbt; Seitenrandeindrücke flach und klein, aber gut erkennbar. Fläche ohne Eindrücke, vor der Mitte mit einer gerade noch wahrnehmbaren Abflachung. Prosternum rau skulpturiert, etwas stärker gewölbt. Mesosternum flach dachförmig; Mittelkiel auf dem Fortsatz schwächer, aber vollständig.

Scutellum fein punktiert. 


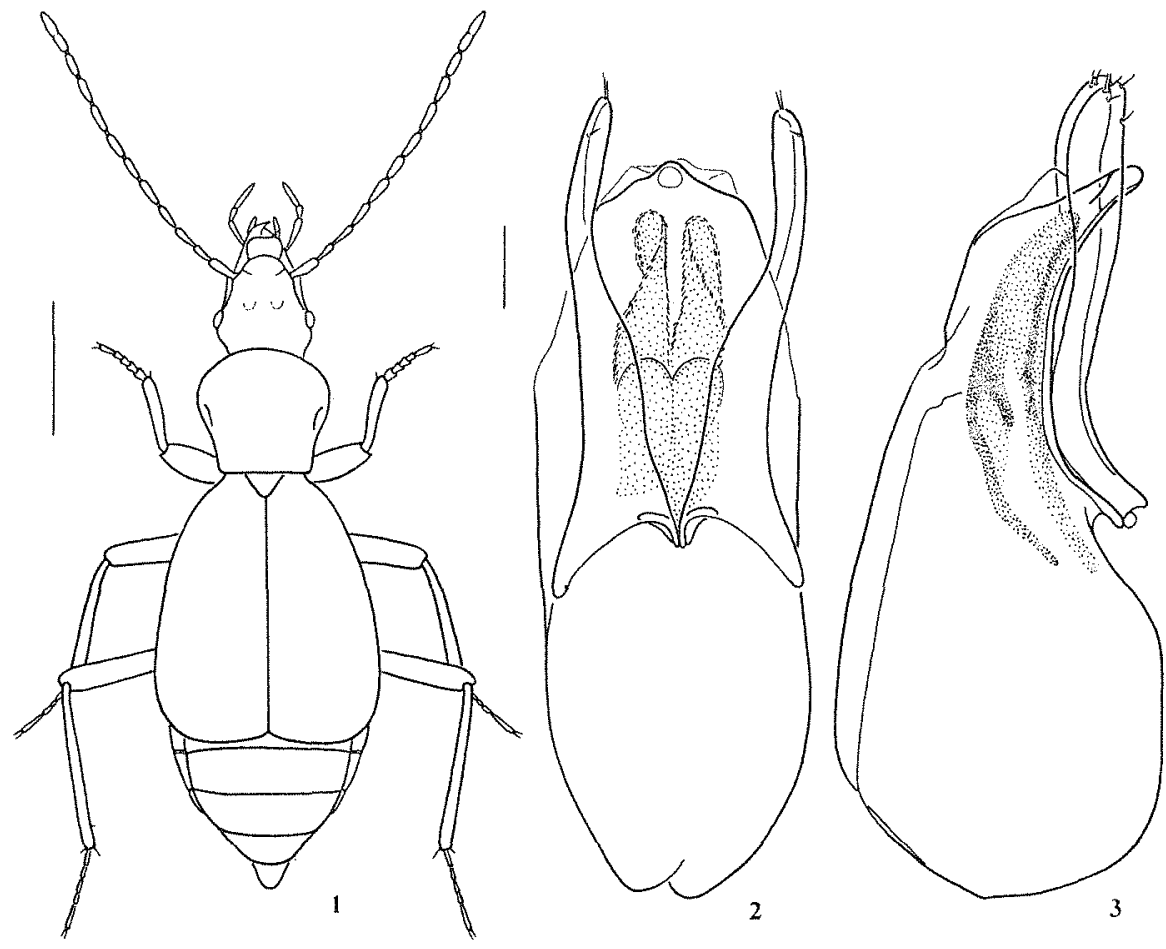

Fig. 1-3: Lesteva brathinoides sp. n. [Maßstab Fig. 1: $1 \mathrm{~mm}$; Fig. 2-3: 0,1 mm] - (1) Habitus [Holotypus]; (2) Aedoeagus, ventral [Holotypus]; (3) Aedoeagus, lateral [Paratypus].

Elytren sehr großflächig und stark gewölbt. Schultern reduziert. Nach hinten sehr stark flachbogig erweitert. Von der breitesten Stelle etwa am Beginn des letzten Drittels nach hinten in gleichmäßigem Bogen verengt. Seitenrand ohne Winkel in den Hinterrand übergehend, dieser bogenförmig zur Naht eingezogen, im inneren Drittel aber fast gerade. Oberfläche in der Vorderhälfte schwach abgeplattet, nach den Seiten stark, fast senkrecht, herab gebogen. Seitenrandung in den beiden vorderen Dritteln von oben sichtbar. Epipleuren bis zum Ende des Metasternums fast von gleicher Breite, dahinter stärker verengt.

Abdomen sehr flach gewölbt; weitgehend unter den Elytren verborgen, nur die Segmente VII und VIII vollständig sichtbar, manchmal auch das Segment VI sichtbar. ${ }^{1}$ Segment V mit sehr schwach ausgebildeten Tomentflecken. Segment VII ohne Fransensaum.

Beine schlank und sehr dünn. Hintertarsen kürzer als die halbe Schienelänge $(0,42)$. Proportionen der Hintertarsen [10 entspricht $0,11 \mathrm{~mm}$ ]: I: 10; II: 7; III: 6; IV: 6; V: 12.

0 : Vordertarsen etwas erweitert, etwa drei Viertel der Breite der Vorderschienen messend. Aedoeagus (Fig. 2-3) breit; Ventralfortsatz sehr kurz und breit. Internalsack aus zahlreichen Setulae gebildet, etwa in der Mitte mit einer charakteristischen doppelbogigen Struktur. Parameren apikal nicht erweitert.

우 Vordertarsen nicht erweitert, nur etwa die Hälfte der Breite der Vorderschienen messend.

${ }^{1}$ Bei dem abgebildete Holotypus (Fig. 1) ist das Abdomen relativ weit sichtbar. Es gibt aber auch bei brathinoides Tiere, die in dieser Beziehung fast dem abgebildeten Paratypus von sajanensis (Fig. 4) entsprechen. In etwas abgeschwächter Form wären die Habitus-Figuren auch umgekehrt möglich gewesen. 


\section{Differentialdiagnose}

Die neue Art ist - ausgenommen Lesteva sajanensis sp. n. - von den anderen Arten der Gattung durch ihren charakteristischen Habitus (Fig. 1) leicht zu unterscheiden. Durch die vollständige Reduktion der Ocellen ist sie von allen Lesteva-Arten zu trennen. Die habituell ähnliche neue Art Lesteva sajanensis aus dem West-Sajan hat zwar kleine Ocellen, diese sind aber deutlich ausgebildet. Lesteva brathinoides hat einen stärker verlängerten Kopf, kleinere Augen und stärker abgeschrägte Schultern. Zu Lesteva sajanensis bestehen auch auffällige Unterschiede in den Proportionen der Hintertarsen, bei brathinoides ist deren Segment I etwas kürzer als das Endglied, bei sajanensis dagegen viel länger als das Endglied, in der Ausbildung der Epipleuren, letztere sind bei brathinoides gleich breit und dann plötzlich verengt, bei sajanensis aber allmählich verengt, und im Bau des Aedoeagus (vergleiche Fig. 2-3 und 5-6). Lesteva brathinoides ist einfarbig rotbraun gefärbt. Lesteva sajanensis hat dagegen einen pechbraun gefärbten Körper und rotbraune Beine.

Lesteva brathinoides ähnelt aber auch den Arten der Gattung Brathinus LECONTE, 1852 (vergleiche die Habitusabbildungen von Brathinus nitidus LECONTE [ARNETT 1961: 367], Brathinus oculatus LEWIS [LAFER 1989: 345] und Brathinus shikokuensis Y. WATANABE \& SATÔ [Y. WATANABE 1990: 249]). Die Trennung von den Brathinus-Arten, zum Vergleich standen nitidus und oculatus zur Verfügung, ist aber leicht: Lesteva brathinoides ist größer, hat viel kleinere Augen und im Gegensatz zu Brathinus einfache Hintertarsen und vor allem nicht die V-förmig angeordneten Scheitel-Vertiefungen der Brathinus-Arten.

\section{Verbreitung}

Es nur der Locus typicus im Massiv Tunkinskiye Gol'tsy bei Arshan im Ost-Sajangebirge

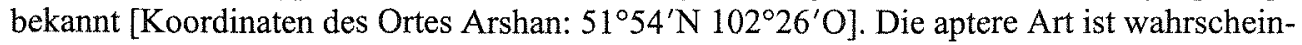
lich ein reliktärer Endemit in diesem Bergmassiv.

\section{Bionomie}

Bekannt sind nur die Sammelumstände: Hochtal zwischen 1700 und $2000 \mathrm{~m}$ ü. NN, Schneefeldrand und blockreicher Steilhang, sowie 2200-2500 $\mathrm{m}$ in der alpinen Zone.

Untersuchte Exemplare: $120^{\pi} 0^{\pi}, 7$ 우오.

Derivatio nominis: Ich wähle den Namen brathinoides (Adjektiv: zusammengesetzt aus dem Gattungsnamen Brathinus und eidés [ähnlich]), weil die neue Art den Brathinus-Arten habituell ähnlich ist. Ich erhielt sie als vermeintliche Brathinus-Art.

\section{Lesteva sajanensis sp.n.}

Fig. 4-6

\section{Typenmaterial}

Holotypus (o⿱): Zap. Sajan, 11-18.VII $\backslash \mathrm{chr}$. [ = chrebet] Kulumys $1985 \backslash 1800 \mathrm{~m}$. perev. [pereval = Gebirgspass] $\backslash \mathrm{V}$. Schilenkov [kyrillisch] $\backslash \backslash$ West Sajan $\backslash$ Kulumys Mts. $\backslash$ alpine Zone $\backslash \backslash$ HOLOTYPUS $\backslash$ Lesteva $\backslash$ sajanensis $\backslash$ Zerche (IGU).

Paratypen ( 4 우 ㅇ): wie Holotypus, aber bei 3 Ex. auf der Unterseite des Etiketts: al'p. lug [= alpine Wiese] $\backslash$ na sklone [= am Abhang] $\backslash S V$ eksp. [= NO-Exposition]; ein Etikett trägt den handschriftlichen Zusatz: u snega [= am Schnee] $\$ Paratypus ... (DEI; IGU; SMNS); (3 o f ): Krasnojarskij kraj \Ermakovskij $r-n$ [= rajon] $\backslash$ Ojskij per. [pereval = Gebirgspass] $8-10 \mathrm{~km} \backslash$ $\mathrm{Ju}[=$ südlich] oz. [= des Sees] Ojskogo $\backslash \mathrm{N} \sim 1800 \mathrm{~m} \backslash 27-28$. VI.90 g. $\backslash \mathrm{Sb}$. V. Morukovitsch [kyrillisch] \I West Sajan \Oiskij pass \Lake Oiskoje \\Paratypus ... (DEI; IGU). 

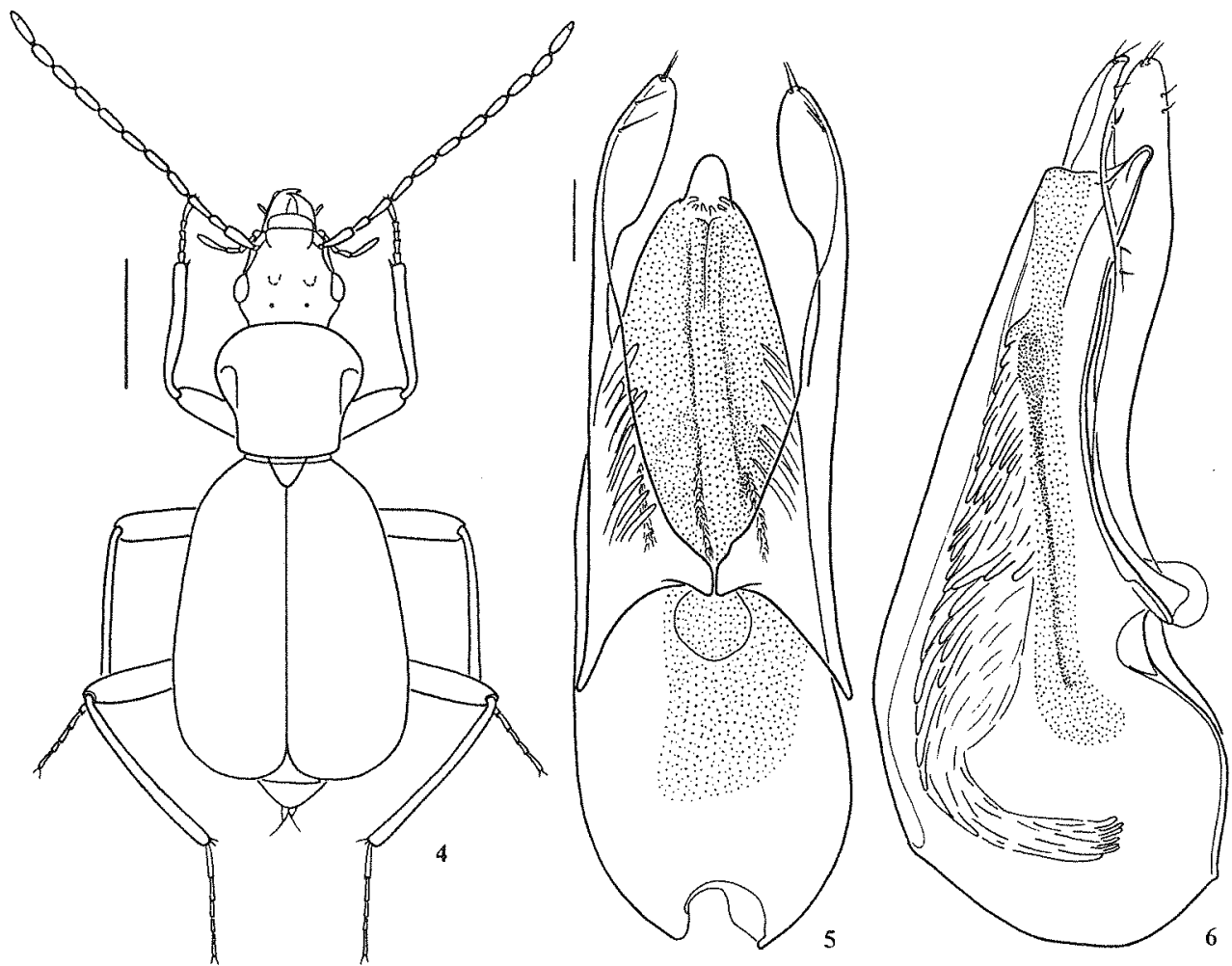

Fig. 4-6: Lesteva sajanensis sp. n. [Maßstab Fig. 4:1 mm; Fig. 5-6: 0,1 mm] - (4) Habitus [Paratypus]; (5) Aedoeagus, ventral [Holotypus]; (6) Aedoeagus, lateral [Holotypus].

\section{Beschreibung}

Maße des Holotypus [in mm]: Kopfbreite 0,95; Kopfmittellänge [ohne Labrum] 0,77; Antennenlänge 2,80; Augenabstand 0,70; Augenlänge 0,22; Schläfenlänge 0,17; Pronotumbreite 1,22; Pronotumlänge 1,11; Elytrenbreite 1,89; Nahtlänge 2,31; Abdomenbreite 1,68.

Körper pechbraun; nur unausgefärbt der Hals, Vorder- und Hinterrand des Pronotums sowie die Naht der Elytren rotbraun abgesetzt. Mundteile, Antennenbasis und Beine immer rotbraun. Kopfseiten, Unterseite des Pronotums und die Epipleuren rotbraun. Ocellen manchmal aufgehellt. Oberseite dicht und fein anliegend hell behaart; Behaarung auf der Stirn wirbelförmig angeordnet. Punktur des Kopfes ziemlich fein und dicht, aber gut sichtbar $(40 \mathrm{x})$, die Zwischenräume etwas kleiner als die Punktdurchmesser, dicht chagriniert. Punktur des Pronotums feiner als die des Kopfes, etwas weniger dicht, glänzend bis sehr schwach chagriniert. Punktur der Elytren deutlich stärker und dichter, aber relativ fein.

Größe: ơ: 5,67 mm; 우: 4,90-5,67 (Mittelwert 5,27 [n=6]).

Kopf gestreckt; Clypeus, Labrum und Mandibeln etwas schnabelförmig nach vorn gerichtet; die größte Länge vom Hinterrand bis zur Mandibelspitze etwas länger als die größte Breite über den Augen $(1,14)$. Scheitel etwas höher als der Hals; eine Halsfurche fehlt oberseits, ist unterseits aber tief, besonders im Bereich der Kehle. Augen ziemlich groß, länger als die Schläfen $(1,29)$ und deutlich vorgewölbt. Ocellen sehr klein; kurz hinter dem Niveau des Augenhinterrandes, voneinander etwas weiter entfernt als jeweils vom Auge $(1,33)$. Stirn- 
gruben hinter dem Niveau des Augenvorderrandes, mäßig tief, aber deutlich. Zwischen der sanft abfallenden Stirn und dem aufgewölbten Clypeus eine tiefe Quermulde. Labrum queroval, Vorderrand sehr flach eingebuchtet. Mandibeln trapezförmig, mit kurzer apikaler Krümmung. Lapialpalpen und Laciniae gestreckt, letztere die Mandibeln aber nicht überragend. Maxillarpalpen schlank; Proportionen der Segmente [10 entspricht $0,11 \mathrm{~mm}$ ]: I: $5 \times 4$; II: 15 x 4; III: $6 \times 4 ; 18 \times 4$. Kehle quer trapezförmig (Länge : Breite $=2: 3,3$ ). Submentum mit ausgeschweiften Seiten (Länge : Breite $=2: 3$ ). Mentum und Submentum durch einen deutlichen stumpfen Winkel voneinander abgesetzt.

Antennen sehr lang und schlank, zurückgelegt fast die Mitte der langen Elytren erreichend. Proportionen der Segmente [10 entspricht 0,11 mm]: I: $25 \times 11$; II: $16 \times 7$; III: 21 x 8,5; IV: 21 x 8; V: $22 \times 8$; VI: $21 \times 9$; VII: $21 \times$ 9; VIII: $23 \times 8$; 5 ;X: $23 \times 9$; X: 21 x 10; XI: $31 \times 10$. Segment XI am Ende des vorderen Drittels nur schwach ringförmig eingeschnürt.

Pronotum im vorderen Drittel am breitesten. Seiten über der breitesten Stelle gleichmäßig gerundet, ohne Andeutung von Vorderwinkeln in den Vorderrand übergehend; Vorderrand im Halsbereich fast gerade. Seitenrand nach hinten stark ausgeschweift verengt. Hinterwinkel etwa rechtwinklig; die Winkel aber nicht scharf, sondern sehr kurz verrundet. Hinterrand gerade bis flach konvex. Seitenrandung sehr fein, unvollständig ausgebildet, vor der breitesten Stelle fehlend. Oberfläche querüber stark gewölbt, aber auch in Längsrichtung deutlich gewölbt; Seitenrandeindrücke flach, aber umfangreich und gut erkennbar. Oberseite ohne Eindrücke und Abflachung. Prosternum weniger rau skulpturiert. Mesosternum flach dachförmig; Mittelkiel in der Hinterhälfte fehlend.

Scutellum fein punktiert.

Elytren sehr großflächig und stark gewölbt; Schultern weitgehend reduziert; nach hinten flachbogig erweitert. Von der größten Breite am Beginn des letzten Drittels nach hinten in gleichmäßigem Bogen verengt. Seitenrand ohne Winkel in den Hinterrand übergehend, dieser bogenförmig zur Naht eingezogen. Oberfläche in der Vorderhälfte schwach abgeplattet, nach den Seiten stark, fast senkrecht, herab gebogen. Seitenrandung nicht von oben sichtbar. Epipleuren von der breitesten Stelle nach hinten gleichmäßig verengt.

Abdomen deutlich gewölbt; weitgehend unter den Elytren verborgen, nur Segment VIII oder nur die Segmente VII und VIII vollständig sichtbar, manchmal auch das Segment VI sichtbar. Segment V ohne Tomentflecke. Segment VII ohne Fransensaum.

Beine schlank und sehr dünn. Hintertarsen länger als die halbe Schienelänge $(0,57)$. Proportionen der Hintertarsen [10 entspricht 0,11 mm]: I: 16; II: 10; III: 8; IV: 6; V: 12.

$\sigma^{7}$ : Vordertarsen etwas erweitert, so breit wie die Vorderschienen [gemessen ohne deren distale Erweiterung]. Aedoeagus (Fig. 5-6) schlank; Apex des Ventralfortsatzes schlank. Parameren sehr gestreckt, zum Apex löffelförmig verbreitert. Internalsack mit kaum sklerotisierten Setae und zahlreichen Setulae.

ๆ: Vordertarsen nicht erweitert, nur etwa die Hälfte der Breite der Vorderschienen erreichend.

\section{Differentialdiagnose}

Lesteva sajanensis $\mathrm{sp} . \mathrm{n}$. ähnelt der Art Lesteva brathinoides $\mathrm{sp} . \mathrm{n}$. Von allen anderen LestevaArten ist sie durch ihren Habitus (Fig. 4) auf den ersten Blick zu unterscheiden. Trotz der äußeren Ähnlichkeit ist die Trennung von brathinoides einfach: Bei sajanensis sind Ocellen vorhanden, während diese bei brathinoides fehlen. Lesteva sajanensis hat sehr gestreckte Hintertarsen; Segment I ist deutlich länger als Segment V. Bei brathinoides sind die Hintertarsen kürzer; Segment I ist etwas kürzer als Segment V. Bei Lesteva sajanensis sind die Epipleuren von ihrer breitesten Stelle nach hinten gleichmäßig verengt, bei brathinoides sind sie gleich breit und dann ziemlich abrupt verengt. 
Die Unterscheidung von den ähnlichen Brathinus-Arten ist leicht: Lesteva sajanensis ist größer, hat weniger prominente Augen und im Gegensatz zu Brathinus einfache Mittel- und Hintertarsen und vor allem nicht die V-förmig angeordneten Scheitel-Vertiefungen der Brathinus-Arten.

\section{Verbreitung}

Die neue Art ist von zwei Lokalitäten im West-Sajangebige bekannt: Chrebet Kulumys [Koordinaten des Ortes Kulumys $52^{\circ} 58^{\prime} \mathrm{N} 92^{\circ} 57^{\prime} \mathrm{O}$ ] und Ojskij pereval [Ojskij Pass] im Ermakovskij Rajon [Name des Ortes: Ermakovskoje $=$ Jermakovskoje $=$ Yermakovskoye]. Die Art ist apter und wahrscheinlich ein reliktärer Endemit dieses Gebirgsmassivs im Süden Sibiriens.

\section{Bionomie}

Es sind nur die Sammelumstände bekannt, einmal ein Pass in $1800 \mathrm{~m}$ Höhe (alpine Zone, auf einer alpinen Wiese am Abhang in NO-Exposition) zum anderen eine Höhe von $\sim 1800 \mathrm{~m}$ und am Schnee gesammelt.

\section{Untersuchte Exemplare: $10^{*}, 7$ 웅.}

Derivatio nominis: Ich wähle den Namen sajanensis (Adjektiv) nach dem Locus typicus im Sajan-Gebirge.

\section{Zur systematischen Stellung der beiden neuen Arten}

Beiden neuen Lesteva-Arten gemeinsam sind der schnabelförmig verlängerte Kopf mit verlängerten Mundteilen, sehr schlanke Antennen und Beine, ein stark gewölbtes und nach hinten stark ausgeschweift verengtes Pronotum, die Reduktion der Hautflügel und der Schultern der Elytren sowie die starke Wölbung der Elytren, die das Abdomen größtenteils einschließen.

Die beiden Arten unterscheiden sich aber in wesentlichen Merkmalen (siehe Tabelle 1).

\section{brathinoides sp. n.}

einfarbig: sowohl der Körper als auch die

Körperanhänge rotbraun

gröber punktiert

Kopf stärker verlängert

Augen klein, nur so lang wie die Schläfen

Ocellen vollständig reduziert ${ }^{2}$

Kehle kaum quer (Länge : Breite $=2: 2,5$ )

\section{sajanensis sp. $\mathrm{n}$.}

zweifarbig: Körper pechbraun, Körperanhänge \pm rotbraun

feiner und dichter punktiert

Kopf weniger stark verlängert

Augen groß, länger als die Schläfen

Ocellen teilweise reduziert, sehr klein

Kehle quer (Länge : Breite $=2: 3,3$ )

${ }^{2}$ Die Reduktion der Ocellen ist bei Omaliinae, vor allem aus Hochgebirgen, nichts Ungewöhnliches. In der Tribus Coryphiini gibt es sie mehrfach unabhängig voneinander, sowohl bei den Arten einer Gattung [Niphetodes, Altaioniphetodes, Ophthalmoniphetodes, Pareudectus, Niphetodops] als auch bei einzelnen Arten innerhalb einer Gattung [Eudectus reductus, Boreaphilus schereri, Hypsonothrus deubeli] (ZERCHE 1990, 1993). 
brathinoides sp. $\mathrm{n}$.

Submentum mit geraden Seiten

Submentum kaum quer (Länge : Breite $=2,2$ : 2,7)

Mentum und Submentum fast in einer Ebene liegend

Segment XI der Antennen in der Mitte stärker abgeschnürt (Autapomorphie)

Seitenrandung des Pronotums vollständig ausgebildet

Seitenrandeindrücke des Pronotums klein

Mesosternum mit vollständigem Mittelkiel

Schultern der Elytren stärker reduziert

Hinterrand der Elytren schräg abgestutzt

Epipleuren bis zum Ende des Metasternums fast von gleicher Breite, dahinter plötzlich verengt

nur die Abdominalsegmente VII und VIII vollständig sichtbar (manchmal auch VI)

Abdominalsegment $\mathrm{V}$ mit sehr schwach ausgebildeten Tomentflecken

Hintertarsen schlank; aber nur 0,42 der Länge der Hinterschienen messend

Segment I der Hintertarsen kürzer als Segment $\mathrm{V}(0,83)$

Aedoeagus gedrungen

Apex des Ventralfortsatzes stumpf zugespitzt und nicht stärker abgesetzt

Parameren kurz, den Ventralfortsatz nur wenig überragend

Parameren apikal nur schwach erweitert sajanensis sp. $\mathrm{n}$.

Submentum mit ausgeschweiften Seiten

Submentum quer (Länge : Breite $=2: 3$ )

Mentum und Submentum durch stumpfen Winkel voneinander abgesetzt

Segment XI am Ende des vorderen Drittels schwach ringförmig eingeschnürt

Pronotum im vorderen Teil ohne Seitenrandung (Autapomorphie)

Seitenrandeindrücke des Pronotums umfangreich

Mittelkiel des Mesosternums in der Hinterhälfte fehlend

Schultern der Elytren schwächer reduziert

Elytren am Hinterrand jeweils einzeln verrundet

Epipleuren von ihrer breitesten Stelle nach hinten gleichmäßig verengt

nur Abdominalsegment VIII oder nur die Segmente VII und VIII vollständig sichtbar (manchmal auch VI)

Abdominalsegment $\mathrm{V}$ ohne Tomentflecke

Hintertarsen sehr schlank; 0,57 der Länge der Hinterschienen messend

Segment I der Hintertarsen länger als Segment $\mathrm{V}(1,33)$

Aedoeagus schlank

Apex des Ventralfortsatzes schlank und deutlich abgesetzt

Parameren lang, den Ventralfortsatz deutlich überragend

Parameren apikal stark erweitert 
Obwohl habituell ungewöhnlich ähnlich - ähnlicher als mit jeder anderen Lesteva-Art - sind die beiden neuen Arten Lesteva brathinoides und Lesteva sajanensis nicht näher miteinander verwandt. Ihre habituelle Ähnlichkeit beruht teilweise auf Konvergenzen, die aus der Reduktion der Hautflügel und der Flugmuskulatur resultieren. Solche Reduktionen sind aus mehreren Gruppen der Omaliinae bekannt, besonders aus der Tribus Coryphiini, wo ein ähnlicher Habitus vielfach konvergent entstanden ist und wo in der phylogenetischen Analyse (ZERCHE 1990) eine auf solchen Reduktionen basierende polyphyletische Gruppierung ausgemerzt werden musste. Einige andere Merkmale stellen Sonderbildungen dar, die zum ähnlichen Habitus beitragen, wie die Verlängerung des Kopfes und der Mundteile und die Vergrößerung und starke Wölbung der Elytren. Diese sind wahrscheinlich eine Folge von ähnlicher Lebensweise. Beide Arten leben nicht an Ufern wie Lesteva-Arten üblicherweise, sondern alpin im Hochgebirge. Beide wurden am Schneerand gesammelt. Ihr auf ähnliche Weise verlängerter Kopf mit jeweils verlängerten Mundteilen kann als ein Indiz für ähnliche Ernährung aufgefasst werden. Beide erinnern etwas an schneckenfressende Arten.

Der Brathinus-ähnliche Habitus ist demnach in der Tribus Anthophagini dreimal unabhängig voneinander evolviert worden, bei den Brathinus-Arten, bei Lesteva brathinoides und bei Lesteva sajanensis.

Wegen des von ihren nächsten Verwandten jeweils stark abweichenden Habitus' der neuen Lesteva-Arten ist es nicht möglich, die jeweilige Schwesterart zu benennen und damit die Stellung im System wahrscheinlich zu machen. Dies muss einer Revision der Gattung vorbehalten bleiben. Es ist aber die Hypothese möglich, dass beide Arten im System weiter voneinander entfernt stehen werden.

Die bisherige Einteilung der Gattung in vier Untergattungen BORDONI (1973) bzw. in drei Untergattungen (ZANETTI 1984, 1987) ist nicht phylogenetisch begründet und wird in dieser Form wahrscheinlich keiner phylogenetischen Prüfung standhalten. Weil deutliche SeitenrandEindrücke des Pronotums vorhanden sind (Fig. 4), wäre Lesteva sajanensis nach BORDONI (1973) und ZANETTI (1987) in die Untergattung Lestevina zu stellen, die aber nicht verfügbar ist (Nomen nudum), weil keine Typusart designiert wurde. Da auch bei Lesteva brathinoides solche Vertiefungen angedeutet sind (Fig. 1), wäre sie ebenfalls dorthin zu stellen. Beide neuen Arten lassen aber jeweils keine mit „Lestevina“ gemeinsamen Merkmale erkennen, die man als Synapomorphien werten könnte. Sie weisen noch nicht einmal Ähnlichkeit mit den dort untergebrachten Arten auf [sicula sicula ERICHSON, 1840; sicula heeri FAUVEL, 1871; foveolata LUZE, 1903; corsica PERRIS, 1869; sbordonii BORDONI, 1973]). Wahrscheinlich sind sie mit keiner der genannten Arten näher verwandt. Da bei den Omaliinae die Existenz von Seitenrand-Gruben auf dem Pronotum die Plesiomorphie und deren Reduktion die Apomorphie darstellt (ZERCHE 1990: 254), würde eine solche unkritisch zusammengewürfelte Gruppierung wahrscheinlich polyphyletisch sein.

\section{Literatur}

ARNETT, R. H. 1961: The beetles of the United States (A manual for identification). Fascicle 25. Brathinidae (LECONTE, 1861). - Washington, D. C.: 367-368.

Bordoni, A. 1973: Lesteva (Lestevina nov.) sbordonii n. sp. della Campania (Col. Staphylinidae). Redia, Firenze 54: 229-234.

LAFER, G. S. 1989: 22. Sem. Brathinidae. - In: LER, P. A. (Hrsg.): Opredelitel' nasekomykh Dal'nego Vostoka SSSR. Tom 3. Zhestkokrylye, ili zhuki. Cast' 1. - Leningrad: 345-346.

LOHSE, G. A. 1962: Die Bedeutung des Flügeldeckendimorphismus bei Lesteva LATR. (Col. Staph.) für die Artsystematik der Omalinen. - Verhandlungen XI. Internationaler Kongreß für Entomologie Wien, 17.-25. August 1960, Bd. I (Sektion I-VI): 81-82. 
WATANABE, Y. 1990: A taxonomic study on the subfamily Omaliinae from Japan (Coleoptera, Staphylinidae). - Mem. Tokyo Univ. Agriculture 31: 59-391.

ZANETTI, A. 1984: Contribution to the knowledge of the Omaliinae from Bulgaria (Col. Staphylinidae). Dt. ent. Z., N. F., Berlin 31 (1-3): 75-82.

ZANETTI, A. 1987: Fauna d'Italia: Coleoptera. Staphylinidae. Omaliinae. - Bologna: XII + 472 S.

ZERCHE, L. 1990: Monographie der paläarktischen Coryphiini (Coleoptera, Staphylinidae, Omaliinae). Berlin: $413 \mathrm{~S}$.

ZERCHE, L. 1993: Monographie der paläarktischen Coryphiini (Coleoptera, Staphylinidae, Omaliinae). Supplementum 1. - Beitr. Ent., Berlin 43 (2): 319-374.

\author{
Anschrift des Verfassers: \\ Dr. LOTHAR ZERCHE \\ Deutsches Entomologisches Institut \\ im Zentrum für Agrarlandschafts- und \\ Landnutzungsforschung (ZALF) \\ Schicklerstraße 5 \\ D - 16225 Eberswalde \\ Deutschland \\ e-mail: zerche@dei-eberswalde.de
}

\title{
Besprechungen
}

SARTORI, M. \& LANDOLT, P.: Atlas de distribution des Emphemeres de suisse (Insecta, Ephemeroptera). - Neuchâtel: Schweizer. Ent. Gesellsch., 1999. - 214 S.: zahlr. Abb., Tab., Verbreitungskart. (Fauna Helvetica; 3). - ISBN 2-88414-014-X. - CHF 35.-

Der Hauptteil des Buches enthält die Verbreitungskarten der 85 bisher für die Schweiz nachgewiesenen Arten, dargestellt auf der Basis eines $5 \mathrm{~km}$-Rasters. Für jede Art werden Informationen zu den folgenden Themen gegeben: Höhenverbreitung (vorwiegend nach den Fundstellen von Larven), Flugzeit (Fangzeitpunkte aller Imagines und Subimagines, aber auch von Larven des letzten Stadiums), Typologie (die von Larven besiedelten Lebensräume), Lebenszyklus, Ökologie, Verbreitung (in der Schweiz und anderen, besonders den angrenzenden europäischen Ländern), Status (zeitliche und räumliche Entwicklung in der Schweiz, wobei nach Möglichkeit ein Vergleich mit älteren Angaben angestrebt wird, um einen eventuellen Rückgang im betrachteten Zeitabschnitt zu dokumentieren).

Die Arbeit basiert auf 1.814 untersuchten Standorten, deren Lage einen guten Deckungsgrad für das Territorium der Schweiz ausweist. Es standen 10.610 Datensätze (Beobachtungen einer Art an einem Ort an einem genauen Datum durch einen Beobachter) und 87.020 identifizierte Individuen (13.175 Adulte oder Subimagines und 73.845 Larven) zur Verfügung, wobei zu bemerken ist, daß von diesen hier verarbeiteten Daten vor 1940 kaum fünf Prozent, in den letzten Jahren (1988-1997) aber über 60 Prozent angefallen sind.

Die Eintagsfliegen mit ihren extrem kurzlebigen, wenig attraktiven Imagines und den auch nicht quasi ambulant zu erbeutenden Larven haben nie jene Zuwendung der Freizeitentomologen gefunden, denen die Faunistik der Schmetterlinge und Käfer in Mitteleuropa ihren heutigen Wissensstand so weitgehend zu danken hat. Die Verarbeitung großer Datenmengen zu wissenschaftlich exakten Resultaten ist heute kein Problem mehr, die Beschaffung dieser Daten allerdings nach wie vor.

Den Autoren und ihren wenigen Helfern gebührt Anerkennung dafür, daß sie beide Aspekte der Aufgabe für die Eintagsfliegen der Schweiz in Angriff genommen und mit diesem Buch ein nützliches Arbeitsmittel auch für weitergehende Forschungsarbeiten vorgelegt haben. 